\title{
Mechanism for gene control by a natural allosteric group I ribozyme
}

\author{
ANDY G.Y. CHEN, ${ }^{1}$ NARASIMHAN SUDARSAN, ${ }^{1,2}$ and RONALD R. BREAKER ${ }^{1,2,3,4}$ \\ ${ }^{1}$ Department of Molecular, Cellular and Developmental Biology, Yale University, New Haven, Connecticut 06520, USA \\ ${ }^{2}$ Howard Hughes Medical Institute, Yale University, New Haven, Connecticut 06520, USA \\ ${ }^{3}$ Department of Molecular Biophysics and Biochemistry, Yale University, New Haven, Connecticut 06520, USA
}

\begin{abstract}
An allosteric ribozyme consisting of a metabolite-sensing riboswitch and a group I self-splicing ribozyme was recently found in the pathogenic bacterium Clostridium difficile. The riboswitch senses the bacterial second messenger c-di-GMP, thereby controlling $5^{\prime}$-splice site choice by the downstream ribozyme. The proximity of this allosteric ribozyme to the open reading frame (ORF) for CD3246 suggests that coenzyme-mediated regulation of splicing controls expression of this putative virulence gene. In the presence of c-di-GMP, the allosteric ribozyme in the CD3246 precursor transcript generates a spliced transcript that retains the riboswitch aptamer. In the absence of c-di-GMP, the ribozyme mediates an alternative GTP attack that results in a truncated transcript (alternative GTP-attack product). Using reporter assays in Escherichia coli, we investigated the difference in gene expression between the spliced product and the alternative GTP-attack product. We provide evidence that CD3246 gene expression is activated if allosteric ribozyme splicing creates a ribosome binding site (RBS) for translation from a UUG start codon. In addition, biochemical and genetic analyses reveal that the riboswitch may further control CD3246 expression by revealing or occluding this newly formed RBS. Therefore, this architecture provides the riboswitch with a mechanism for extended regulation after splicing has occurred or as a backup mechanism for suppression of translation in the event of misregulated splicing.
\end{abstract}

Keywords: c-di-GMP; noncoding RNA; riboswitch; second messenger; self-splicing

\section{INTRODUCTION}

Cyclic di-guanosyl-5' -monophosphate (c-di-GMP) is a ubiquitous bacterial second messenger that, among many physiological processes, regulates virulence, motility, and biofilm formation (Hengge 2009; Römling and Simm 2009). In many bacteria, c-di-GMP enhances the biosynthesis of biofilm components (Karatan and Watnick 2009) and stimulates the transition from motile to sessile lifestyles (Hengge 2009). Diguanylate cyclases (DGC) synthesize c-di-GMP from two molecules of guanosine- $5^{\prime}$-triphosphate (GTP), while c-diGMP-specific phosphodiesterases (PDE) degrade c-di-GMP into $5^{\prime}$-phosphoguanylyl- $\left(3^{\prime}-5^{\prime}\right)$-guanosine (pGpG) (Hengge 2009; Schirmer and Jenal 2009).

The major downstream gene control targets for this second messenger had largely remained mysterious (Jenal 2004) until the identification of two classes of c-di-GMP-sensing riboswitches called class I (Sudarsan et al. 2008) and class II

\footnotetext{
${ }^{4}$ Corresponding author.

E-mail ronald.breaker@yale.edu.

Article published online ahead of print. Article and publication date are at http://www.rnajournal.org/cgi/doi/10.1261/rna.2757311.
}

(Lee et al. 2010). Riboswitches are regulatory elements that are often located upstream of coding sequences (Roth and Breaker 2009). A typical riboswitch contains a conserved, highly structured aptamer domain that binds a specific metabolite and an expression platform that regulates gene expression using mechanisms such as transcription attenuation and ribosome binding site (RBS) sequestration (Roth and Breaker 2009). The functions of many of the gene products whose expression is controlled by these two riboswitch classes span the physiological processes known to be affected by c-di-GMP. However, many other riboswitch-associated genes code for proteins of unknown function (Sudarsan et al. 2008; Lee et al. 2010). These observations suggest that far more is yet to be learned about the roles of c-di-GMP in cells and the mechanisms this bacterial second messenger uses to regulate diverse biological processes.

In Clostridium difficile 630, a class II c-di-GMP riboswitch regulates the splicing of an $\sim 600$-nt group I self-splicing ribozyme (Lee et al. 2010), which lies between the riboswitch and the coding sequence of CD3246, a putative virulence gene. Previous analyses (Lee et al. 2010) of the noncoding region of the $C D 3246$ precursor mRNA revealed that a ligandbound aptamer stabilizes the $5^{\prime}$-splice site substructure for 
attack by GTP. This arrangement promotes the formation of a spliced transcript in which the aptamer of the $5^{\prime}$ exon is fused to the coding sequence of the $3^{\prime}$ exon. In contrast, a ligandfree aptamer exposes an alternative GTP attack site and causes removal of nearly the entire $5^{\prime}$-untranslated region ( $5^{\prime}$ UTR) of the precursor mRNA (Fig. 1A; Lee et al. 2010). Although molecular engineering methods have been used to create numerous allosteric ribozymes (Thompson et al. 2002; Breaker 2002), this C. difficile allosteric ribozyme implicated in gene regulation constitutes the first natural example.

The mechanism for alternative splicing activity by this allosteric ribozyme has been established through in vitro biochemical analysis (Lee et al. 2010). However, the mechanism by which allosteric ribozyme action results in gene regulation has remained to be validated in vivo. It was hypothesized that the spliced product and the alternative GTPattack product will express the downstream ORF at different amounts (Lee et al. 2010). According to this hypothesis, the alternative GTP-attack product lacks an RBS, whereas the spliced product carries a newly formed and canonical RBS sequence (AGGAGG) located at the perfect distance upstream of a noncanonical UUG start codon (Fig. 1A; Lee et al. 2010). In the present study, we set out to assess the validity of this proposed gene control mechanism. Furthermore, since the riboswitch aptamer remains intact after splicing, we sought to determine whether second messenger binding may continue to contribute to gene regulation in the spliced product even after its role in splicing control is complete.

A series of reporter assays using various constructs was performed in a surrogate organism (Escherichia coli) to investigate the difference in gene expression between the spliced product and the alternative GTP-attack product. Our findings are consistent with the original hypothesis for gene control. In addition, a combination of biochemical and genetic analyses reveals that the riboswitch aptamer continues to regulate gene expression even after splicing, which provides a backup mechanism to regulate CD3246 translation. Thus, the $C D 3246$ riboswitch aptamer controls gene expression at the level of pre-mRNA splicing and translation initiation.

\section{RESULTS AND DISCUSSION}

\section{Ribozyme splicing generates an active translation start site}

To assess the functional role of allosteric ribozyme splicing in translation control, we fused the respective $5^{\prime}$-UTR sequences of the precursor transcript (Pre), the spliced product (Spl), and the alternative GTP-attack product (Alt) to a lacZ reporter gene (Fig. 1A), and quantified $\beta$-galactosidase expression in E. coli (Fig. 1B). As predicted by our model, both the Pre and Alt RNA transcripts do not exhibit reporter gene expression. For Pre, this result suggests that the allosteric ribozyme does not splice efficiently in E. coli
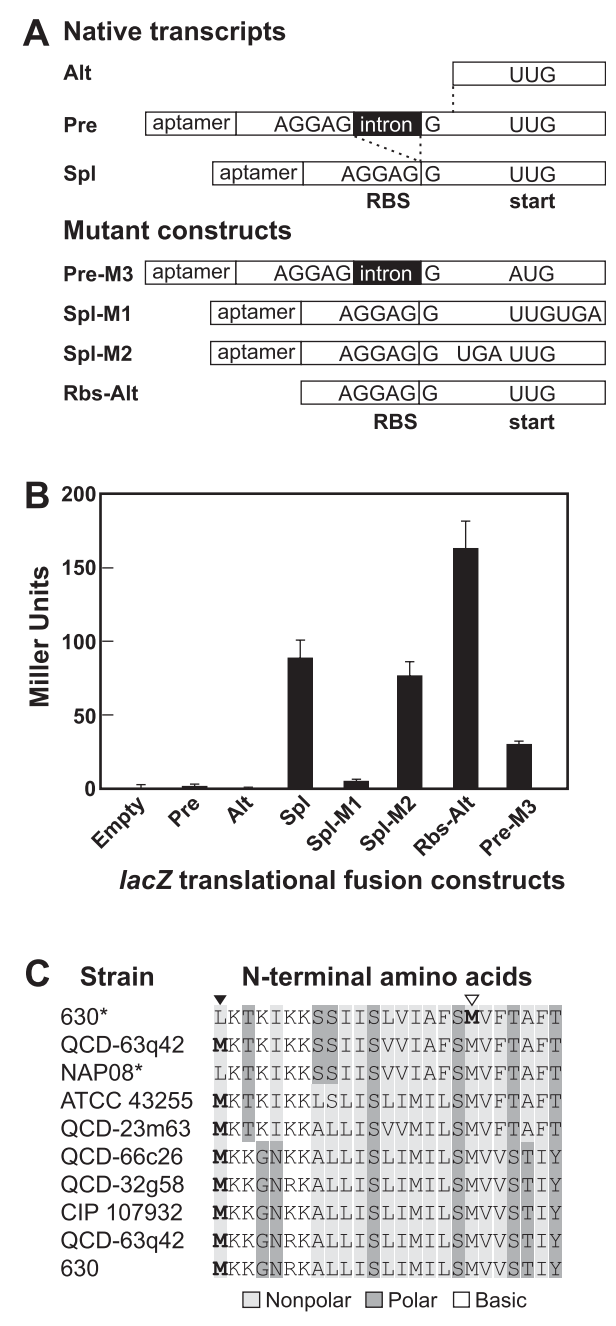

FIGURE 1. Gene expression of various reporter constructs based on the allosteric ribozyme from $C$. difficile. (A) Features of native CD3246 transcripts and several mutant constructs. Native transcripts include the precursor CD3246 transcript (Pre), the alternative GTP-attack product (Alt), and the product resulting from allosteric self-splicing (Spl). Mutant constructs are derivatives of Pre, Spl, and Alt. The group I ribozyme intron is shaded black. Dashed lines depict splicing and the alternative GTP-attack events. (B) $\beta$-Galactosidase activity in E. coli transformed with lac $Z$ translational fusion constructs. A strain containing empty (no insert) pRS414 was used as the control. Three independent experiments were performed; bars report standard error. $(C)$ Alignment of the initial polypeptide sequences encoded by CD3246 in C. difficile 630 (top sequence) and CD3246 homologs in various $C$. difficile strains. Some strains have multiple homologs. Methionine residues predicted from the genome annotations to reside at the $\mathrm{N}$ termini are in bold, and asterisks indicate strains with potentially incorrect annotation. Arrowheads indicate the proposed UUG start codon of CD3246 (closed; tentatively depicted as leucine) and the annotated AUG start codon (open).

to produce detectable levels of $\beta$-galactosidase. Alt shows no $\beta$-galactosidase activity, presumably due to the lack of an RBS. In contrast, Spl is translationally active, suggesting that the product of allosteric splicing carries a start codon and RBS configuration that is active for translation initiation.

Our interpretation of the above observations was further assessed by examining the reporter gene activity when fused 
to several mutant constructs based on the Spl or Alt RNAs. For example, we propose that $\mathrm{Spl}$ is active because it carries an RBS sequence (AGGAGG) that is perfectly complementary to the RBS recognition sequence (CCUCCU) present in 10 out of 11 mRNA binding sites in $16 \mathrm{~S}$ rRNAs of $C$. difficile 630 (Sebaihia et al. 2006). We inserted this same RBS sequence in front of the alternative GTP-attack product (RbsAlt) (Fig. 1A) and found that the resulting construct gained robust translational activity (Fig. 1B). These data support the hypothesis that the allosteric ribozyme controls CD3246 expression at least in part by constructing the RBS sequence only when sufficient c-di-GMP is present.

For $C$. difficile strain 630, a UUG triplet resides $6 \mathrm{nt}$ downstream from the ribozyme-created RBS sequence. Although this sequence does not match the typical AUG start codon, the amino acid sequence immediately downstream from this UUG is conserved in other organisms. This noncanonical start codon aligns perfectly with the start codons from this same gene in other organisms, and it resides in-frame with the remainder of the ORF (Fig. 1C). However, the existing genome annotation assigns the start codon to be an AUG codon located further downstream. To pinpoint the start codon of CD3246, we placed stop codons near the UUG triplet in Spl fusion constructs (Fig. 1A). Introducing a stop codon immediately downstream from the putative noncanonical UUG start codon (Spl-M1) eliminates expression, whereas introducing a stop codon immediately upstream of this UUG (Spl-M2) has negligible effect on expression (Fig. 1B). This result demonstrates that UUG indeed is the start codon for the CD3246 protein.

Intriguingly, the vast majority of annotated, noncanonical start codons in C. difficile 630 use AGGAGG for ribosome binding (data not shown). In addition, multiple $C$. difficile strains contain the tandem riboswitch-ribozyme architecture in CD3246 homologs, and all of these strains use the UUG start codon. To test whether the conservation of UUG at the start position is critical for tight regulation of gene expression, we replaced UUG with AUG and constructed Pre-M3 (Fig. 1A). As a stronger start codon, AUG uses a suboptimal RBS and causes leaky expression from the unspliced precursor transcript (Fig. 1B). Therefore, allosteric ribozyme function creates an optimal juxtaposition of RBS and noncanonical start codon to activate gene expression. Collectively, these analyses validate the hypothesis that, in the presence of c-di-GMP, the self-splicing activity of the ribozyme generates an RBS sequence to promote translation from a UUG codon, thereby regulating gene expression. In the absence of c-di-GMP, however, the alternative GTP attack by the ribozyme generates a truncated mRNA that lacks an RBS for this same codon.

\section{The allosteric ribozyme may require specific protein cofactors to facilitate splicing}

As noted above, expression of the full-length $5^{\prime}$-UTR precursor (Pre) fused to lac $Z$ yields negligible amounts of $\beta$-galactosidase expression (Fig. 1B). To detect any spliced product in E. coli strains transformed with Pre, we extracted total RNA from overnight bacterial cultures and performed reverse transcriptase (RT)-PCR with DNA oligonucleotides flanking the splice sites (Supplemental Table S1). We were only able to detect the precursor transcript but not the spliced product (data not shown). This is likely due to the inability of the $C$. difficile allosteric ribozyme to undergo efficient splicing in E. coli. In addition, some group I ribozymes require the formation of a ribonucleoprotein complex to facilitate splicing (Lambowitz et al. 1999). Therefore, E. coli may lack the specific protein cofactor that promotes efficient ribozyme self-splicing in vivo. Since large, multidomain RNAs are likely to misfold (Jackson et al. 2006), the C. difficile ribozyme may be trapped in non-native states when placed in a surrogate host.

\section{The riboswitch aptamer in the spliced product retains ligand-binding function in vitro}

Splicing transfers the entire riboswitch aptamer upstream of the newly formed RBS sequence. Due to its close proximity to the RBS, we speculated that the aptamer may continue to regulate gene expression via an RBS-occlusion mechanism. To assess the ability of the riboswitch to sense and respond to c-di-GMP, we constructed a 126-nt RNA based on the spliced product, hereafter termed $126 \mathrm{Spl}$ (Fig. 2A). We subjected $126 \mathrm{Spl}$ RNAs to in-line probing analysis either with or without c-di-GMP (Fig. 2B). In-line probing exploits the natural instability of RNA to elucidate secondary structure characteristics of RNA molecules (Soukup and Breaker 1999; Regulski and Breaker 2008).

In the presence of c-di-GMP, $126 \mathrm{Spl}$ exhibits reduced scission in the pseudoknot (Fig. 2A,B, nucleotides 51-57 and 79-87), as expected for proper aptamer function. Most notably, the ligand causes increased scission in the area of nucleotides 98 through 106 (Fig. 2B, region 1) and reduced scission in the area of nucleotides 90 to 96 (Fig. 2B, region 2). Region 1 contains part of the RBS sequence and has basepairing potential with nucleotides located in the $5^{\prime}$ shoulder of the P1 stem (Figs. 2A, 3A). In contrast, region 2 overlaps the nucleotides of the $3^{\prime}$ shoulder of $\mathrm{P} 1$, which must form the P1 stem when c-di-GMP is bound to the aptamer (Lee et al. 2010). These in-line probing data suggest that an alternative anti-RBS stem forms instead of the aptamer P1 stem if c-di-GMP is absent, which would lead to repression of the adjoining ORF.

\section{The riboswitch continues to regulate translation in the spliced product in vivo}

To test the RBS-occlusion mechanism in vivo, we used three different $E$. coli strains with deletions in PDE genes $(\Delta d o s P, \Delta g m r, \Delta y h j K)$ in an effort to alter the in vivo concentration of c-di-GMP. The dos $P$, gmr , and $y h j K$ genes 


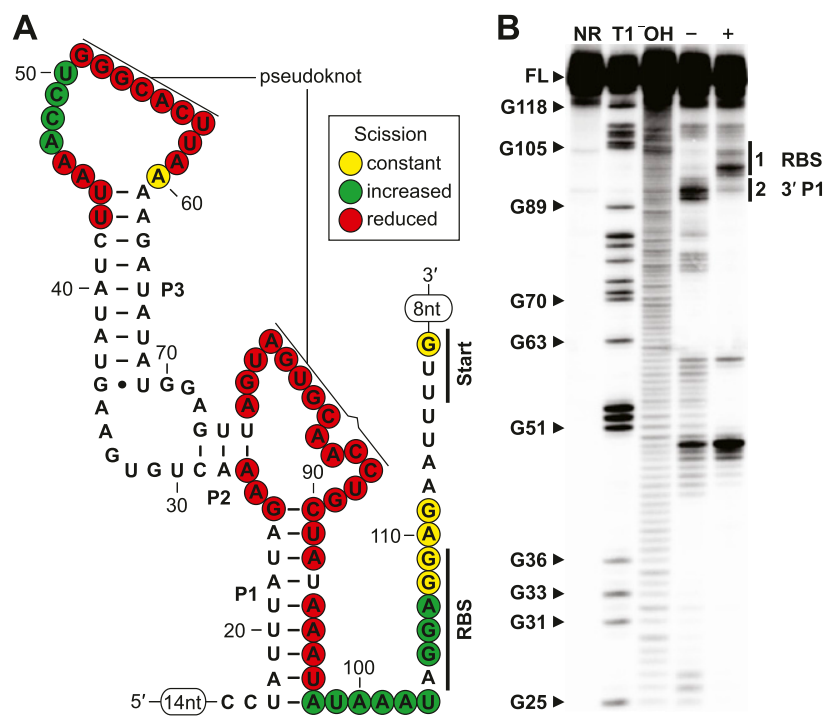

FIGURE 2. The spliced mRNA product exhibits anti-RBS pairing potential in vitro. $(A)$ Sequence and secondary structure model of the 126 Spl RNA. RBS and UUG start codon sequences are labeled. Colored circles identify patterns of spontaneous RNA cleavage, with red and green indicating nucleotide linkages that undergo reduced or increased cleavage, respectively, on the addition of saturating concentrations of c-di-GMP $(10 \mu \mathrm{M})$. Data are derived from the image depicted in $B$. (B) In-line probing analysis of the 126 Spl RNA, showing nucleotides 25 to 126. Lanes are labeled as follows: no reaction (NR), partial digest with RNase T1 (T1; cleaves after every G residue), partial digest with alkali ( ${ }^{-} \mathrm{OH}$; cleaves after all nucleotides), and incubation in the absence $(-)$ or presence $(+)$ of $10 \mu \mathrm{M}$ c-di-GMP. Arrowheads indicate the full-length (FL) RNA band and other bands representing RNase T1 cleavage after selected G residues. Vertical bars highlight nucleotides that become unstructured (region 1, RBS) and structured (region 2, 3' P1) in the presence of c-di-GMP.

encode enzymes ( $y h j K$ function is putative) that catalyze c-di-GMP-specific degradation (Weber et al. 2006; Tuckerman et al. 2009). Since these PDEs degrade c-di-GMP, which otherwise signals biofilm formation (Karatan and Watnick 2009), the PDE-deletion strains should form more biofilm than the wild-type strain.

Using crystal violet to quantify biofilm in these E. coli strains, we found that the extent of biofilm formation occurs as follows: wild-type $<\Delta \operatorname{dos} P \leq \Delta y h j K<\Delta g m r$ (Fig. 3B). Notably, the $\Delta g m r$ strain forms fivefold more biofilm than wild type, likely due to more accumulation of c-diGMP. These results are consistent with the finding that $g m r$ is constitutively expressed, while $\operatorname{dos} P$ and $y h j K$ are highly expressed only in stationary phase (Sommerfeldt et al. 2009).

We found that Pre does not give higher reporter activity even in these PDE-deletion strains (Fig. 3C). This supports the idea that the level of c-di-GMP concentration in E. coli is likely not the major cause for the inefficient splicing. In addition, we expected the riboswitch in Spl to form a stabilized, ligand-bound conformation under high c-di-GMP conditions. Consistent with this notion, $\beta$-galactosidase expression in strains that bear the Spl reporter construct were as follows: wild-type $\leq \Delta \operatorname{dos} P \leq \Delta y h j K<\Delta g m r$ (Fig. 3C). This finding suggests that cellular c-di-GMP modulates translation of the spliced product, even after its effect on allosteric ribozyme splicing has been executed.

Additional riboswitch reporter fusions were designed to evaluate the proposed alternative base-pairing model for translation control. Spl-M4 carries a mutation that disrupts the anti-RBS stem and favors the formation of the aptamer P1 stem (Fig. 3A). All four strains bearing the Spl-M4 reporter construct exhibit high $\beta$-galactosidase expression compared with the wild-type Spl construct, regardless of cellular c-di-GMP concentrations (Fig. 3C). This is consistent with the putative role for nucleotide 100 in suppressing gene expression. In contrast, Spl-M5 carries an additional mutation at nucleotide 21 that restores the anti-RBS stem but disrupts the aptamer P1 stem (Fig. 3A). All four strains bearing the Spl-M5 reporter construct exhibit low levels of reporter gene expression (Fig. 3C), suggesting that c-di-GMP can no longer activate this construct. Spl-M6 has been modified with a 9-nt insertion that forms a new RBS sequence that should be unaffected by conformational changes in the aptamer (Fig. 3A). This insertion nullifies the differences among the strains, and every strain exhibits high $\beta$-galactosidase expression (Fig. 3C). Along with in-line probing data (Fig. 2), the above results reveal that the riboswitch can regulate translation in the spliced product.
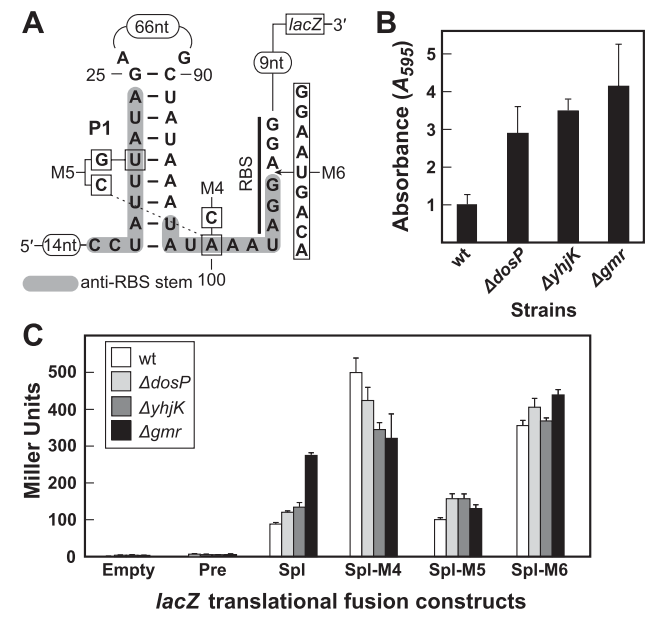

FIGURE 3. The aptamer can regulate translation in the spliced transcript in vivo via an RBS-occlusion mechanism. (A) Spl-derived lac $Z$ fusion constructs with the aptamer $\mathrm{P} 1$ pairing (as shown) and the proposed anti-RBS conformation (shaded gray). The positions of mutations in Spl-M4, -M5, and -M6 are shown. (B) Quantitation of biofilm formed by overnight cultures of wild-type (wt) and mutant strains of $E$. coli using crystal violet. The attachment of crystal violet to the biofilm was measured at $A_{595}$ and normalized to the amount of biofilm formed by wild-type $E$. coli. Three independent experiments were performed; bars report standard error. (C) $\beta$-Galactosidase activity from wild-type (wt) and mutant strains of E. coli transformed with Spl-derived lacZ fusion constructs. A strain bearing pRS414 was used as the control. Three independent experiments were performed; bars report standard error. 


\section{CONCLUSIONS}

The confirmed allosteric and gene-control activities of the tandem riboswitch-ribozyme from $C$. difficile demonstrates how self-splicing ribozymes, although often considered "selfish" elements, may confer benefits to their cellular hosts. Given that the splicing activity of group I ribozymes depends on guanosine or related derivatives such as GTP, this C. difficile system may regulate gene expression by sensing both c-di-GMP and compounds in the pool of guanosine derivatives in the cell. It seems likely that CD3246 expression will be reduced when GTP concentration is insufficient for splicing, regardless of the concentration of c-di-GMP. During the transition from exponential phase to stationary phase, the cellular concentration of c-di-GMP increases (Hengge 2009), and the concentration of GTP drops accordingly (Buckstein et al. 2008). Therefore, there may be a narrow time frame in which both c-di-GMP and GTP are sufficient for high expression of $C D 3246$.

Moreover, the ability of the spliced RNA to continue to respond to changing $\mathrm{c}$-di-GMP concentrations provides a second level of regulatory control in addition to the control of ribozyme splicing (Fig. 4). Previous in vitro analysis showed that $\sim 5 \%$ of precursor transcripts yield spliced prod-

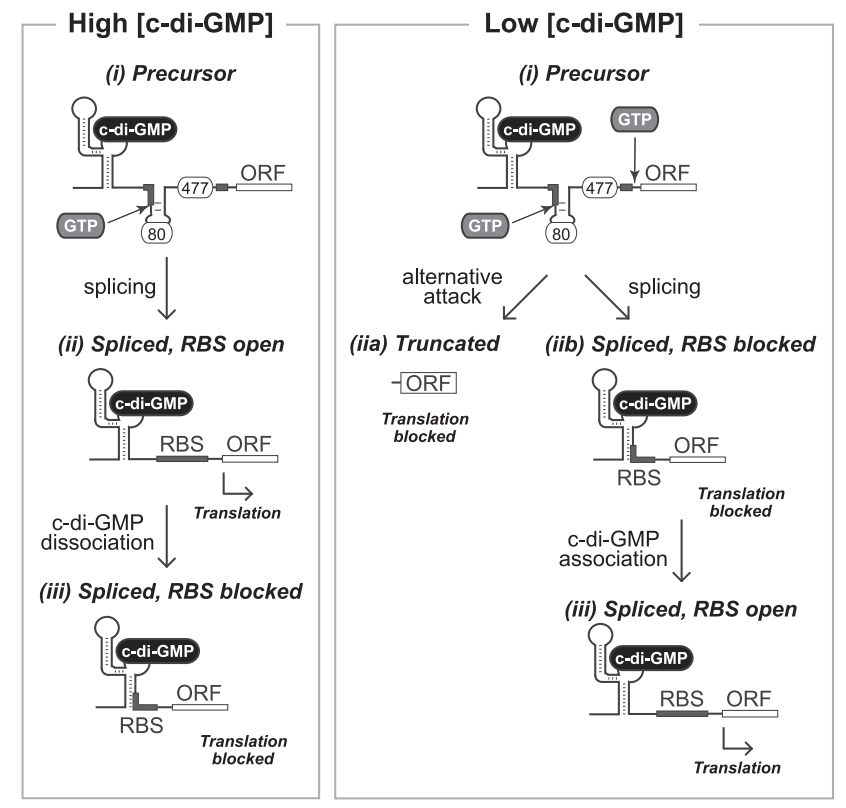

FIGURE 4. Gene regulation by an allosteric ribozyme in $C$. difficile 630. (Left) Precursor allosteric ribozyme (i) activity in the presence of both c-di-GMP and GTP yields spliced RNA that carries an accessible RBS (ii). Subsequent dissociation of c-di-GMP could switch gene expression off by occluding the RBS (iii). Large (AGGAG) and small (G) dark gray boxes designate the RBS sequence components that the group I ribozyme splices together. (Right) In the absence of c-di-GMP, precursor allosteric ribozyme (i) activity primarily yields truncated RNAs (iia) that are not expressed. If splicing sometimes occurs (iib), the absence of c-di-GMP results in RBS occlusion and gene suppression. Subsequent association of c-di-GMP (iii) can activate gene expression by making the RBS accessible. ucts in the complete absence of c-di-GMP (Fig. 4; Lee et al. 2010). In such erroneously spliced transcripts, the riboswitch can serve as a backup mechanism that ensures translational inhibition when c-di-GMP concentrations are low (Fig. 4, right). Moreover, changing concentrations can alter gene expression even after splicing has occurred, since dissociation from or association to the aptamer by the second messenger will alter RBS accessibility. Therefore, the tandem riboswitchribozyme permits control over two stages of the gene expression pathway. The riboswitch may act as security against leaky splicing or may be used to extend the time frame over which c-di-GMP can influence expression of the downstream gene.

\section{MATERIALS AND METHODS}

\section{Bacterial strains}

The wild-type E. coli K-12 strain used in this study was BW25113 [F-, $\Delta$ (araD-araB)567, $\Delta$ lacZ4787(::rrnB-3), lambda ${ }^{-}$rph-1, $\Delta$ (rhaDrhaB)568, hsdR514]. Mutant strains JW1278-1 (Dgmr-722::kan), JW1484-4 ( $\Delta$ dos-782::kan), and JW5943-1 ( $\Delta$ yhjK-784::kan) are isogenic derivatives of BW25113. These mutant strains were selected on kanamycin $(50 \mu \mathrm{g} / \mathrm{mL})$. All bacterial strains were obtained from the Coli Genetics Stock Center (Yale University) and cultured at $37^{\circ} \mathrm{C}$ in LB medium. Plasmids were introduced into these strains via electroporation using a Gene Pulser Xcell (Bio-Rad) and were selected on LB agar plates containing carbenicillin $(50 \mu \mathrm{g} / \mathrm{mL})$.

\section{Reporter-fusion constructs}

The DNA for the Pre construct was amplified from the C. difficile 630 genomic DNA by using PCR, and this construct was used as a template to prepare other constructs. The DNA sequences of Spl, Alt, and their mutant derivatives were generated by one-step or twostep PCR reactions using DNA oligonucleotides (Sigma-Aldrich) (see Supplemental Table S1 for all sequences). Each of these constructs was fused to a constitutive promoter and inserted immediately upstream of the E. coli lacZ gene using EcoRI and BamHI sites in pRS414 (Simons et al. 1987). All constructs were transformed into NEB 5- $\alpha$ E. coli cells (New England Biolabs) according to the manufacturer's instructions, and the sequences were confirmed by the Keck DNA Sequencing Facility prior to conducting assays.

\section{$\beta$-Galactosidase assays}

Bacterial strains transformed with reporter constructs were grown overnight in LB medium containing appropriate antibiotics. Cultures were diluted (1:1000) in $2 \mathrm{~mL}$ of LB and grown for $4 \mathrm{~h}$. $\beta$-Galactosidase activity was measured in triplicate as previously described (Miller 1992; Sudarsan et al. 2006). LB medium without bacterial inoculation was used to establish background values.

\section{Biofilm quantitation}

Biofilm formation was quantified in triplicate as described previously (O’Toole et al. 1999). Briefly, E. coli strains bearing pRS414 were cultured in 5-mL borosilicate glass tubes (VWR LabShop) vertically and statically. Bacteria were inoculated in $2 \mathrm{~mL}$ of $\mathrm{LB}$ 
(initial $\mathrm{OD}_{600}=0.05$ ) and incubated for $24 \mathrm{~h}$ at room temperature. The cultures were then decanted and rinsed with deionized water three times. Afterward, $2 \mathrm{~mL}$ of $0.1 \%(\mathrm{w} / \mathrm{v})$ aqueous crystal violet was added to the tubes and incubated for $20 \mathrm{~min}$. The tubes were then vigorously rinsed with deionized water three times. Associated dye was then solubilized using ethanol-acetone (80\%:20\%) and quantified at $A_{595}$. A tube with LB medium without bacterial inoculation was used to establish background values.

\section{In-line probing}

In-line probing was performed as described previously (Regulski and Breaker 2008). The DNA sequence of $126 \mathrm{Spl}$ was fused to the T7 promoter using DNA oligonucleotides (Sigma-Aldrich) (Supplemental Table S1). The $126 \mathrm{Spl}$ RNA was prepared by in vitro transcription using T7 RNA polymerase in a solution containing $80 \mathrm{mM}$ HEPES-KOH ( $\mathrm{pH} 7.5$ at $23^{\circ} \mathrm{C}$ ), $24 \mathrm{mM} \mathrm{MgCl}_{2}, 2 \mathrm{mM}$ spermidine, and $40 \mathrm{mM}$ dithiothreitol (DTT). The transcription products were subjected to denaturing (8 M urea) 6\% polyacrylamide gel electrophoresis (PAGE) for purification. The RNA transcripts were dephosphorylated using alkaline phosphatase (Roche Diagnostics) and radiolabeled with $\left[\gamma-{ }^{32} \mathrm{P}\right]$ ATP using T4 polynucleotide kinase (New England Biolabs) according to the manufacturers' instructions. The radiolabeled RNA was purified using $6 \%$ denaturing PAGE. The purified RNA was subsequently incubated in the absence or presence of $10 \mu \mathrm{M} \mathrm{c}$-di-GMP for $45 \mathrm{~h}$ at room temperature in a solution containing $50 \mathrm{mM}$ Tris- $\mathrm{HCl}(\mathrm{pH} 8.3$ at $23^{\circ} \mathrm{C}$ ), $20 \mathrm{mM} \mathrm{MgCl}_{2}$, and $100 \mathrm{mM} \mathrm{KCl}$. Spontaneous scission products were resolved by denaturing 10\% PAGE. Gels were dried and visualized using a Molecular Dynamics PhosphorImager.

\section{SUPPLEMENTAL MATERIAL}

Supplemental material is available for this article.

\section{ACKNOWLEDGMENTS}

We thank members of the Breaker laboratory for helpful discussions, and particularly Dr. Elaine Lee for preliminary work in generating pRS414 constructs. This work was supported by NIH and the Howard Hughes Medical Institute.

Received March 29, 2011; accepted August 19, 2011.

\section{REFERENCES}

Breaker RR. 2002. Engineered allosteric ribozymes as biosensor components. Curr Opin Biotechnol 13: 31-39.

Buckstein MH, He J, Rubin H. 2008. Characterization of nucleotide pools as a function of physiological state in Escherichia coli. J Bacteriol 190: 718-726.
Hengge R. 2009. Principles of c-di-GMP signalling in bacteria. Nat Rev Microbiol 7: 263-273.

Jackson SA, Koduvayur S, Woodson SA. 2006. Self-splicing of a group I intron reveals partitioning of native and misfolded RNA populations in yeast. RNA 12: 2149-2159.

Jenal U. 2004. Cyclic di-guanosine-monophosphate comes of age: a novel secondary messenger involved in modulating cell surface structures in bacteria? Curr Opin Microbiol 7: 185-191.

Karatan E, Watnick P. 2009. Signals, regulatory networks, and materials that build and break bacterial biofilms. Microbiol Mol Biol Rev 73: 310-347.

Lambowitz AM, Caprara M, Zimmerly S, Perlman P. 1999. Group I and group II ribozymes as RNPs: Clues to the past and guides to the future. In The RNA world (ed. RF Gesteland et al.), pp. 451-485. Cold Spring Harbor Laboratory Press, Cold Spring Harbor, NY.

Lee ER, Baker JL, Weinberg Z, Sudarsan N, Breaker RR. 2010. An allosteric self-splicing ribozyme triggered by a bacterial second messenger. Science 329: 845-848.

Miller JH. 1992. A short course in bacterial genetics: Handbook. Cold Spring Harbor Laboratory Press, Cold Spring Harbor, NY.

O'Toole GA, Pratt LA, Watnick PI, Newman DK, Weaver VB, Kolter R. 1999. Genetic approaches to study of biofilms. Methods Enzymol 310: 91-109.

Regulski EE, Breaker RR. 2008. In-line probing analysis of riboswitches. Methods Mol Biol 419: 53-67.

Römling U, Simm R. 2009. Prevailing concepts of c-di-GMP signaling. Contrib Microbiol 16: 161-181.

Roth A, Breaker RR. 2009. The structural and functional diversity of metabolite-binding riboswitches. Annu Rev Biochem 78: 305-334.

Schirmer T, Jenal U. 2009. Structural and mechanistic determinants of c-di-GMP signaling. Nat Rev Microbiol 7: 724-735.

Sebaihia M, Wren BW, Mullany P, Fairweather NF, Minton N, Stabler $\mathrm{R}$, Thomson NR, Roberts AP, Cerdeño-Tárraga AM, Wang H. 2006. The multidrug-resistant human pathogen Clostridium difficile has a highly mobile, mosaic genome. Nat Genet 38: 779-786.

Simons RW, Houman F, Kleckner N. 1987. Improved single and multicopy lac-based cloning vectors for protein and operon fusions. Gene 53: 85-96.

Sommerfeldt N, Possling A, Becker G, Pesavento C, Tschowri N, Hengge R. 2009. Gene expression patterns and differential input into curli fimbriae regulation of all GGDEF/EAL domain proteins in Escherichia coli. Microbiology 155: 1318-1331.

Soukup GA, Breaker RR. 1999. Relationship between internucleotide linkage geometry and the stability of RNA. RNA 5: 1308-1325.

Sudarsan N, Hammond MC, Block KF, Welz R, Barrick JE, Roth A, Breaker RR. 2006. Tandem riboswitch architectures exhibit complex gene control functions. Science 314: 300-304.

Sudarsan N, Lee ER, Weinberg Z, Moy RH, Kim JN, Link KH, Breaker RR. 2008. Riboswitches in eubacteria sense the second messenger cyclic di-GMP. Science 321: 411-413.

Thompson KM, Syrett HA, Knudsen SM, Ellington AD. 2002. Group I aptazymes as genetic regulatory switches. BMC Biotechnol 2: 21-32.

Tuckerman JR, Gonzalez G, Sousa EHS, Wan X, Saito JA, Alam M, Gilles-Gonzalez MA. 2009. An oxygen-sensing diguanylate cyclase and phosphodiesterase couple for c-di-GMP control. Biochemistry 48: 9764-9774.

Weber H, Pesavento C, Possling A, Tischendorf G, Hengge R. 2006. Cyclic-di-GMP-mediated signalling within the $\sigma^{\mathrm{s}}$ network of Escherichia coli. Mol Microbiol 62: 1014-1034. 

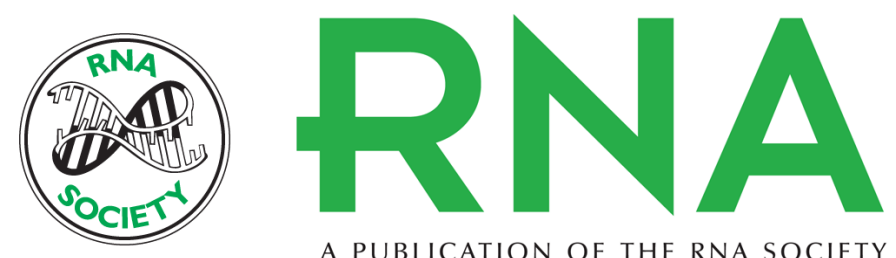

A PUBLICATION OF THE RNA SOCIETY

\section{Mechanism for gene control by a natural allosteric group I ribozyme}

Andy G.Y. Chen, Narasimhan Sudarsan and Ronald R. Breaker

RNA 2011 17: 1967-1972 originally published online September 29, 2011

Access the most recent version at doi:10.1261/rna.2757311

Supplemental

Material

References

Open Access

License Freely available online through the RNA Open Access option.

Freely available online through the RNA Open Access option.
http://rnajournal.cshlp.org/content/suppl/2011/08/31/rna.2757311.DC1

This article cites 21 articles, 7 of which can be accessed free at: http://rnajournal.cshlp.org/content/17/11/1967.full.html\#ref-list-1

Receive free email alerts when new articles cite this article - sign up in the box at the top right corner of the article or click here. 